\title{
Multiplex PCR for detection of the Vibrio genus and five pathogenic Vibrio species with primer sets designed using comparative genomics
}

\author{
Hyun-Joong Kim, Ji-Oh Ryu, Shin-Young Lee, Ei-Seul Kim and Hae-Yeong Kim*
}

\begin{abstract}
Background: The genus Vibrio is clinically significant and major pathogenic Vibrio species causing human Vibrio infections are V. cholerae, V. parahaemolyticus, V. vulnificus, V. alginolyticus and V. mimicus. In this study, we screened for novel genetic markers using comparative genomics and developed a Vibrio multiplex PCR for the reliable diagnosis of the Vibrio genus and the associated major pathogenic Vibrio species.

Methods: A total of 30 Vibrio genome sequences were subjected to comparative genomics, and specific genes of the Vibrio genus and five major pathogenic Vibrio species were screened. The designed primer sets from the screened genes were evaluated by single PCR using DNAs from various Vibrio spp. and other non-Vibrio bacterial strains. A sextuplet multiplex PCR using six primer sets was developed to enable detection of the Vibrio genus and five pathogenic Vibrio species.

Results: The designed primer sets from the screened genes yielded specific diagnostic results for target the Vibrio genus and Vibrio species. The specificity of the developed multiplex PCR was confirmed with various Vibrio and non-Vibrio strains. This Vibrio multiplex PCR was evaluated using 117 Vibrio strains isolated from the south seashore areas in Korea and Vibrio isolates were identified as Vibrio spp., V. parahaemolyticus, V. vulnificus and V. alginolyticus, demonstrating the specificity and discriminative ability of the assay towards Vibrio species.

Conclusions: This novel multiplex PCR method could provide reliable and informative identification of the Vibrio genus and major pathogenic Vibrio species in the food safety industry and in early clinical treatment, thereby protecting humans against Vibrio infection.
\end{abstract}

Keywords: Identification, PCR, Vibrios, V. cholerae, V. parahaemolyticus, V. vulnificus, V. alginolyticus, V. mimicus, Comparative genomics

\section{Background}

The Vibrio genus, which consists of more than 30 species, includes a number of major foodborne pathogens. Eleven of these Vibrio species are known to be human pathogens causing toxigenic cholera and other infections (vibriosis). $V$. cholerae, $V$. parahaemolyticus, $V$. vulnificus, $V$. alginolyticus and $V$. mimicus are pathogens of note in the clinical microbiology and food safety fields [1-6]. Vibrio are ubiquitous in halophilic marine environments and the consumption of raw or undercooked contaminated seafood causes human infections worldwide [4, 6-8]. The Cholera

\footnotetext{
* Correspondence: hykim@khu.ac.kr

Institute of Life Sciences \& Resources and Graduate School of Biotechnology, Kyung Hee University, Yongin 446-701, Republic of Korea
}

and Other Vibrio Illness Surveillance System (COVIS) of the Centers for Disease Control and Prevention (CDC) and the World Health Organization (WHO) considers pathogenic Vibrio species to be a public health threat and annually reports the number of human infections during Vibrio outbreaks (vibriosis by pathogenic Vibrio species including $V$. parahaemolyticus, $V$. vulnificus, $V$. alginolyticus, $V$. mimicus and other Vibrio species; Cholera due to toxigenic $V$. cholerae) $[2,9,10]$.

Molecular biological DNA-based diagnostic methods, especially polymerase chain reaction (PCR), have been studied and developed for accurate and rapid identification of Vibrio spp. These methods provide advantages relative to and/or that complement standard microbiological culture-based methods $[8,11]$. In early studies, 
PCR diagnostic methods were developed separately for each Vibrio species using specific expected virulence factor genes as genetic markers, including the cholera toxin (ctx) gene for $V$. cholerae and $V$. mimicus $[8,12]$, the thermostable direct hemolysin $(t d h)$ gene and the thermostable direct hemolysin-related hemolysin (trh) gene for $V$. parahaemolyticus [13-15], and the cytotoxin-haemolysin $(v v h A)$ gene for $V$. vulnificus $[16,17]$. Recently, several genes, such as the regulatory gene tox $R$ and the housekeeping genes $\operatorname{atp} A$, rpoB, and $d n a J$, have been suggested as novel genetic markers for use in PCR methods to complement the diagnosis of Vibrio species [3, 18-21]. Multiplex PCR methods for diagnosis of major pathogenic Vibrio species have also been developed [3, 21-25], and modified PCR methods with DNA-DNA hybridization or other technologies have been attempted for the accurate and rapid detection of Vibrio species [26-28].

In our previous studies, comparative genomics was used to screen for genetic markers for designing specific primer sets for Salmonella spp. and other pathogenic bacteria. The selected genetic markers were successfully used in the identification of Salmonella enterica serovars and other pathogenic bacteria, reflecting the potential application of genomics and bioinformatics for the detection and identification of foodborne pathogens [29-32]. Through the Vibrio genome projects, 21 Vibrio genomes have been completely sequenced and were available at the National Center for Biotechnology Information (NCBI) in 2012 (more than 100 draft Vibrio genome projects are also available or in progress). The original purpose of this study was to develop novel genetic markers, which would enable reliable and comprehensive diagnostics for Vibrio species. We employed an 'in silico' approach utilizing comparative genomics between genome sequences of Vibrio spp., thereby differentiating our method from previously reported Vibrio PCR methods. Specific expected genes for the Vibrio genus and 5 Vibrio species, which were chosen based on their significance as human pathogens as well as based on sequences being available through the NCBI, were screened using comparative genomics. Ultimately, a sextuplet Vibrio multiplex PCR was developed from the screened specific genes and the utility of this assay was evaluated.

\section{Results}

Screening of Vibrio genus- and species-specific genes using comparative genomics and the design of primer sets

To screen for Vibrio genus-specific genes, a total of 4832 gene sequences from $V$. parahaemolyticus RIMD 2210633 [GenBank: NC_004603.1, NC_004605.1] were compared with each representative genome sequence (fna file) from 4 Vibrio species. Ultimately, 1256 genes expected to be present in the Vibrio genus were selected based on the outputs of the Basic Local Alignment Search Tool (BLAST) program that indicated their relatively high matched DNA length (bp), thereby eliminating genes of low homology within the Vibrio genus. These genes were also compared to the non-redundant (nr) database of NCBI to eliminate genes that were highly matched with other biological sources. A total of 38 genes, which resulted low homology (less than $30 \mathrm{bp}$ size that matched sequences in the $n r$ database), were chosen and were compared individually to the $\mathrm{nr}$ and the microbial genome databases of the NCBI BLAST web site to again confirm their specificity in the Vibrio genus [33]. Finally, 2 genes (ATP synthase subunit alpha, C3281450-3279879 [GenBank: NC_004603.1] and recombinase A, C2694352-2693309 [GenBank: NC_004603.1]) were selected for the design and evaluation of primer sets for Vibrio genus-specific detection.

To screen for specific genes in each of the Vibrio species, the coding region sequences representing each of 5 Vibrio species were analyzed using the BLAST program to compare them against Vibrio genome sequences without the genome sequences of the targeted Vibrio species. The outputs of the BLAST program were analyzed by screening a number of genes (between $400 \sim 650$ genes) to eliminate highly homologous genes within the Vibrio genus and to select those with matching under $<100$ (or 50) bp. The screened genes were then compared to the $\mathrm{nr}$ database of NCBI to select genes specific to the targeted Vibrio species with relatively low homology matching a length $<22$ (or 23) bp of DNA. An appropriate number of specific expected genes (or DNA fragments) within the target Vibrio species were selected as follows: 59 genes from $V$. parahaemolyticus RIMD 2210633, 94 genes from $V$. cholerae O1 biovar El Tor str. N16961, 134 genes from $V$. vulnificus YJ016, 23 genes from $V$. alginolyticus $12 \mathrm{G} 01$ and 39 genes from $V$. mimicus MB-451. These selected genes were evaluated for their gene sizes and also were individually compared using the microbial genome database (complete and draft genome) on the NCBI BLAST web site [33]. In total, 15 primer sets (3 primer sets of each Vibrio species) expected to be specific for each species were designed. The designing of primers sets was considered by melting temperature (Tm), PCR product size and the regions of conserved (or varied) sequence in the target genes within Vibrio species.

\section{The specificity of designed primer sets for the Vibrio genus and species}

The specificity of the designed primer sets was evaluated by conventional single PCR using various Vibrio species and other representative pathogenic bacteria, as listed in Table 1. In spite of the expected specificity of the genes screened through comparative genomics, 5 of 17 primer sets (one primer set from each of the five targeted Vibrio species) resulted in unexpected PCR results (i.e., amplification of non-targeted Vibrio species, unexpected PCR 
Table 1 Bacterial strains used in this study

\begin{tabular}{|c|c|c|c|c|c|}
\hline Bacteria Strains & Source & $\operatorname{Lane}^{a}$ & Bacteria Strains & Source & Lane \\
\hline Vibrio cholerae & NCCP 13589 & 1 & Escherichia coli EHEC (O157:H7) & ATCC 43890 & 23 \\
\hline Vibrio alginolyticus & ATCC $^{C} 17749$ & 2 & Escherichia coli O157:H7 & ATCC 43894 & 24 \\
\hline Vibrio mimicus & ATCC 33653 & 3 & Escherichia coli & ATCC 35150 & 25 \\
\hline Vibrio parahaemolyticus & ATCC 27969 & 4 & Escherichia coli & ATCC 11775 & 26 \\
\hline Vibrio parahaemolyticus & ATCC 33844 & 5 & Listeria monocytogenes & ATCC 19113 & 27 \\
\hline Vibrio parahaemolyticus & ATCC 17802 & 6 & Listeria seeligeri & ATCC 35967 & 28 \\
\hline Vibrio vulnificus & ATCC 33815 & 7 & Listeria innocua & ATCC 33090 & 29 \\
\hline Vibrio vulnificus & ATCC 27562 & 8 & Salmonella enterica serovar Typhimurium & ATCC 19585 & 30 \\
\hline Vibrio vulnificus & ATCC 33147 & 9 & Salmonella enterica serovar Typhi & ATCC 33459 & 31 \\
\hline Vibrio vulnificus & ATCC 33814 & 10 & Salmonella enterica serovar Enteritidis & ATCC 4931 & 32 \\
\hline Vibrio aestuarianus & ATCC 35048 & 11 & Salmonella enterica serovar Gallinarum & ATCC 9184 & 33 \\
\hline Vibrio algoinfesta & $\mathrm{KCCM}^{\mathrm{d}} 40861$ & 12 & Salmonella enterica serovar Pullorum & ATCC 9120 & 34 \\
\hline Vibrio diazotrophicus & ATCC 33466 & 13 & Shigella flexneri & ATCC 12022 & 35 \\
\hline Vibrio fluvialis & ATCC 33809 & 14 & Shigella flexneri 2a strain $2457 \mathrm{~T}$ & ATCC 700930 & 36 \\
\hline Vibrio harveyi & ATCC 35084 & 15 & Shigella dysenteriae & ATCC 13313 & 37 \\
\hline Vibrio harveyi & ATCC 14126 & 16 & Shigella boydi & ATCC 8700 & 38 \\
\hline Vibrio mediterranei & ATCC 43341 & 17 & Shigella boydi & ATCC 9905 & 39 \\
\hline Vibrio salmonicida & ATCC 43839 & 18 & Shigella sonnei & ATCC 25931 & 40 \\
\hline enteroinvensive E. coli (EIEC) & ATCC 43893 & 19 & Staphylococcus aureus & ATCC 29737 & 41 \\
\hline enteroaggregative E. coli (EAEC) & NCCP 14039 & 20 & Stapylococcus epidermidis & ATCC 14990 & 42 \\
\hline enteropathogenic E. coli (EPEC) & NCCP 14038 & 21 & Stapylococcus haemolyticus & ATCC 29970 & 43 \\
\hline enterotoxigenic E. coli (ETEC) & NCCP 14037 & 22 & Yersinia enterocolitica & ATCC 29913 & 44 \\
\hline
\end{tabular}

aLane, This number indicates the lane number in Fig. 1

${ }^{\mathrm{b}} \mathrm{NCCP}$, National Culture Collection for Pathogens

'ATCC, American Type Culture Collection

${ }^{d}$ KCCM, Korean Culture Center of Microorganisms

product size, or no PCR amplification), while the other 12 primer sets were shown to be specific, based on their targeting of the Vibrio genus and species (data not shown). For Vibrio genus-specific detection, two primer sets, C3281450 F36-R518 and C2694352 F46-R734, amplified specific 483-bp and 689-bp fragments with Vibrio strains and the primer set C2694352 F46-R734 was selected for multiplex PCR design. One primer set for each of the Vibrio species was selected based on their constant intensity of amplified PCR product and the lack of non-specific bands upon agarose gel analysis. For further confirmation of the PCR specificity of each primer set against the targeted Vibrio genus or species, the PCR products were sequenced and the sequence was compared with the sequence of the original target gene. All sequences were as expected (data not shown).

\section{Specificity, sensitivity and multi-detection ability of Vibrio multiplex PCR}

Based on the evaluation of each primer set by single conventional PCR, one of each of the specific primer sets for the Vibrio genus, V. parahaemolyticus, V. cholerae, V. vulnificus, $V$. alginolyticus, and $V$. mimicus was selected based on their Tm, the intensity of the amplified PCR product and PCR product size. A multiplex PCR reaction, including an internal amplification control (IAC, $104 \mathrm{bp)}$ ), was constructed. Table 2 details their sources, primer concentrations, primer sequences and the expected PCR product sizes. The amount of the constructed plasmid used for the IAC and the concentration of each primer set were adjusted based on the amplified band intensities resulting from repeat multiplex PCR reactions using genomic DNAs from Vibrio strains. The discriminative ability and specificity of this Vibrio multiplex PCR was evaluated with various genomic DNAs of Vibrio and other non-Vibrio strains, as shown in Fig. 1. This multiplex PCR was designed to amplify 7 bands (lane P, Fig. 1) including the IAC, thereby enabling identification of the Vibrio genus and five major pathogenic Vibrio species, as shown in Fig. 1 (lanes $1 \sim$ 10: the specifically targeted Vibrio species; lanes $11 \sim 18$ : Vibrio spp. other than the specifically targeted Vibrio species). No false positive bands were amplified with other non-Vibrio bacterial strains using this multiplex PCR, which further 
Table 2 Primer pairs for Vibrio multiplex PCR designed and used in this study and their sources

\begin{tabular}{|c|c|c|c|c|c|c|}
\hline Primer name & Source of gene ${ }^{a}$ & $\begin{array}{l}\text { Target genus } \\
\text { or species }\end{array}$ & $\begin{array}{l}\text { PCR product } \\
\text { size (bp) }\end{array}$ & $\begin{array}{l}\text { Final primer } \\
\text { Conc. }(\mu \mathrm{M})\end{array}$ & Primer sequences $\left(5^{\prime}-3^{\prime}\right)^{b}$ & Protein of target gene \\
\hline VP $1155272 \mathrm{~F}$ & $\begin{array}{l}\text { NC_004605.1 } \\
\text { (c1155272-1154856) }\end{array}$ & Vibrio parahaemolyticus & 297 & 0.24 & $\begin{array}{l}\text { 5' AGCTT ATTGG CGGTT } \\
\text { TCTGT CGG }\end{array}$ & $\begin{array}{l}\text { hypothetical protein } \\
\text { VPA1095 }\end{array}$ \\
\hline VP $1155272 R$ & & & & & $\begin{array}{l}5^{\prime} \text { CKCAA GACCA AGAAA } \\
\text { AGCCG TC }\end{array}$ & \\
\hline VC C634002 F & $\begin{array}{l}\text { NC_002506.1 } \\
\text { (c634002-633547) }\end{array}$ & Vibrio cholerae & 154 & 0.24 & $\begin{array}{l}\text { 5' CAAGC TCCGC ATGTC } \\
\text { CAGAA GC }\end{array}$ & $\begin{array}{l}\text { hypothetical protein } \\
\text { VCA0694 }\end{array}$ \\
\hline VC C634002 R & & & & & $\begin{array}{l}\text { 5' GGGGC GTGAC GCGAA } \\
\text { TGATT }\end{array}$ & \\
\hline W 2055918 F79 & $\begin{array}{l}\text { NC_005139.1 } \\
(2055918-2056664)\end{array}$ & Vibrio vulnificus & 484 & 0.4 & $\begin{array}{l}\text { 5' CAGCC GGACG TCGTC } \\
\text { CATTT TG }\end{array}$ & $\begin{array}{l}\text { hypothetical protein } \\
\text { W2055 }\end{array}$ \\
\hline W 2055918 R & & & & & $\begin{array}{l}\text { 5' ATGAG TAAGC GTCCG } \\
\text { ACGCG T }\end{array}$ & \\
\hline VA $1198230 \mathrm{~F}$ & $\begin{array}{l}\text { NZ_CH902589.1 } \\
(1198230-1198616)\end{array}$ & Vibrio alginolyticus & 199 & 0.1 & $\begin{array}{l}\text { 5' ACGGC ATTGG AAATT } \\
\text { GCGAC TG }\end{array}$ & $\begin{array}{l}\text { whole genome shotgun } \\
\text { sequence }\end{array}$ \\
\hline VA $1198230 \mathrm{R}$ & & & & & $\begin{array}{l}5^{\prime} \text { TACCC GTCTC ACGAG } \\
\text { CCCAA G }\end{array}$ & \\
\hline VM C727581F & $\begin{array}{l}\text { NZ_ADAF01000001.1 } \\
(\mathrm{c} 727581-726859)\end{array}$ & Vibrio mimicus & 249 & 0.8 & $\begin{array}{l}\text { 5' ATAAA GCGGG CTTGC } \\
\text { GTGCA }\end{array}$ & $\begin{array}{l}\text { contig } 43 \text {, whole genome } \\
\text { shotgun sequence }\end{array}$ \\
\hline VM C727581R & & & & & $\begin{array}{l}\text { 5' GATTT GGRAA AATCC } \\
\text { KTCGT GC }\end{array}$ & \\
\hline VG C2694352 F46 & $\begin{array}{l}\text { NC_004603.1 } \\
\text { (c2694352-2693309) }\end{array}$ & Vibrio genus & 689 & 1 & $\begin{array}{c}\text { 5' GTC ARA TTG AAA ARC } \\
\text { ART TYG GTA AAG G }\end{array}$ & recombinase $\mathrm{A}$ \\
\hline VG C2694352 R734 & & & & & $\begin{array}{l}\text { 5' ACY TTR ATR CGN GTT } \\
\text { TCR TTR CC }\end{array}$ & \\
\hline
\end{tabular}

${ }^{a}$ Reference sequence number of chromosomes in GenBank at the NCBI and position of gene

${ }^{\mathrm{b}}$ Mixed base: $\mathrm{K}=\mathrm{G}+\mathrm{T} ; \mathrm{R}=\mathrm{A}+\mathrm{G} ; \mathrm{Y}=\mathrm{C}+\mathrm{T} ; \mathrm{N}=\mathrm{A}+\mathrm{C}+\mathrm{G}+\mathrm{T}$


Fig. 1 Results of the Vibrio multiplex PCR and its specificity using various genomic DNAs of Vibrio and other pathogenic bacterial type strains. M: 100-bp DNA ladder, P: Mixture of Vibrio cholerae KCDC 13589, V. alginolyticus ATCC 17749, V. mimicus ATCC 33653, V. parahaemolyticus ATCC 27969, V. vulnificus ATCC 33815, lanes $1 \sim$ lane 44: The information of type strains in each lane is indicated in Table 1, NT: no template. Vspp, Vibrio genus; W, V. vulnificus; VP, V. parahaemolyticus; VM, V. mimicus; VA, V. alginolyticus; VC, V. cholerae 
demonstrates the specificity and discriminative ability of this Vibrio multiplex PCR. Additionally, the limit of detection (LOD) and evaluation using genomic DNA combinations from various Vibrio species were performed to confirm the sensitivity and multi-detection ability of the Vibrio multiplex PCR. Analysis of agarose gel electrophoresis loaded with $5 \mu \mathrm{l}$ from a $25 \mu \mathrm{l}$-PCR showed the detection limit to be between $5 \times 10^{3}$ and $5 \times 10^{2}$ copies of Vibrio genomic DNA in a 25 -cycle PCR and between $5 \times$ $10^{2}$ and $5 \times 10^{1}$ copies in a 30 -cycle PCR, as shown in Additional file 1 . The multi-detection ability was evaluated using a combination of genomic DNAs from Vibrio species and resulted in the specific amplification of fragments of the expected size and number with the different Vibrio species (Additional file 2).

\section{Evaluation of multiplex PCR with Vibrio isolates from seashore areas in Korea}

Isolated strains of Vibrio collected by the Ministry of Food and Drug Safety (MFDS), the Busan Metropolitan city Institute of Health \& Environment (BMIHE), and our laboratory from the south seashore areas in Korea using microbiological culture-based procedures were used to evaluate the specificity/applicability of our multiplex PCR assay. A total of 117 isolates were subjected to Vibrio multiplex PCR and all of the Vibrio isolates showed positive results with the Vibrio genus-specific primer set (689 bp, VG C2694352 F46-R734), as shown in Table 3 and Fig. 2 (representative isolates are shown in Fig. 2). Among the 117 isolates, 94 strains resulted in a positive reaction, without any non-specific bands, with the Vibrio species-specific band identifying each as $V$. parahaemolyticus, $V$. vulnificus or $V$. alginolyticus, thereby reflecting the clear discriminative ability of the Vibrio multiplex PCR. No isolates of $V$. cholerae or $V$. mimicus were found in the environmental samples using this Vibrio multiplex PCR. Interestingly, 23 isolates were found to be Vibrio spp. other than the five targeted pathogenic Vibrio species, with a single amplification of the VG C2694352 F46-R734 primer set. Sequencing of the PCR products of the Vibrio isolates confirmed the expected amplification of each target gene, supporting the accuracy and specificity of the Vibrio multiplex PCR (data not shown). As a counterpart method to confirm the results of this Vibrio multiplex PCR with the Vibrio isolates, the representative Vibrio isolates in Fig. 2 were analyzed using Matrix-Assisted Laser-Desorption Ionization Time-of-Flight Mass Spectrometry (MALDI-TOF MS) and were identified same with the result of Vibrio multiplex PCR as shown in Table 4. [three strains of Vibrio species (lanes 2, 5 and 7) were identified as Vibrio mytili and Vibrio fortis; other nine strains were identified same Vibrio species as Vibrio alginolyticus (lanes 1, 3, 6 and 9) Vibrio parahaemolyticus (lanes 4 and 10) and Vibrio vulnificus (lanes 8 and 11)].

\section{Discussion}

The reliability of phenotype-based identification methods, which are laborious and time-consuming procedures that include microbiological culture-based identification, for detection of the Vibrio genus and specific Vibrio species has been questioned due to variations in the biochemical characteristics within the genus $[4,34]$. In particular, the rapid differential diagnosis of clinically important $V$. cholerae from other Vibrio species is essential and economical and rapid diagnosis of $V$. cholerae is critical in mitigating the spread of Vibrio during an outbreak, as well as aiding in epidemic-preventing surveillance [12, 35-37]. Also, the current gold standard for laboratory diagnosis of cholera has been issued due to the lengthy culturing time required on selective growth media (TCBS is the only proven selective and ideal media for selective isolation and purification of Vibrio spp.) $[4,8,12]$. As an alternative to culture-based identification, molecular biological methods, especially PCR, have been developed for the detection and identification of pathogenic

Table 3 Number of isolated Vibrio strains from local areas of Korea and their multiplex PCR results

\begin{tabular}{|c|c|c|c|c|c|c|c|}
\hline \multirow[t]{3}{*}{ Area of sampling } & \multirow{3}{*}{$\begin{array}{l}\text { \# of } \\
\text { sample }\end{array}$} & \multicolumn{6}{|c|}{ Results of each specific primer set in multiplex $P C R^{a}$} \\
\hline & & $\overline{V P}$ & VC & W & VA & VM & Vibrio genus \\
\hline & & VP C1155272 F-R & VC C634002 F-R & W 2055918 F79-R & VA $1198230 \mathrm{~F}-\mathrm{R}$ & VM C727581 F-R & VG C2694352 F46-R734 \\
\hline Yeosu (by MFDS ${ }^{b}$ ) & 20 & & & 20 & & & 20 \\
\hline Busan & 8 & 2 & & 5 & 1 & & 8 \\
\hline Busan (by BMIHE') & 34 & 34 & & & & & 34 \\
\hline Geoje & 14 & & & & 4 & & 14 \\
\hline Jinhae & 34 & 5 & & & 21 & & 34 \\
\hline Chungmu & 7 & & & & 2 & & 7 \\
\hline Total & 117 & 41 & 0 & 25 & 28 & 0 & 117 \\
\hline
\end{tabular}

${ }^{\mathrm{V} V P}, \mathrm{~V}$. parahaemolyticus; $\mathrm{VC}, \mathrm{V}$. cholerae; $\mathrm{V}, \mathrm{V}$. vulnificus; $\mathrm{VA}, \mathrm{V}$. alginolyticus; $\mathrm{VM}, \mathrm{V}$. mimicus

${ }^{\mathrm{b}}$ MFDS, Ministry of Food and Drug Safety in South Korea

${ }^{C}$ BMIHE, Busan Metropolitan city Institute of Health \& Environment 


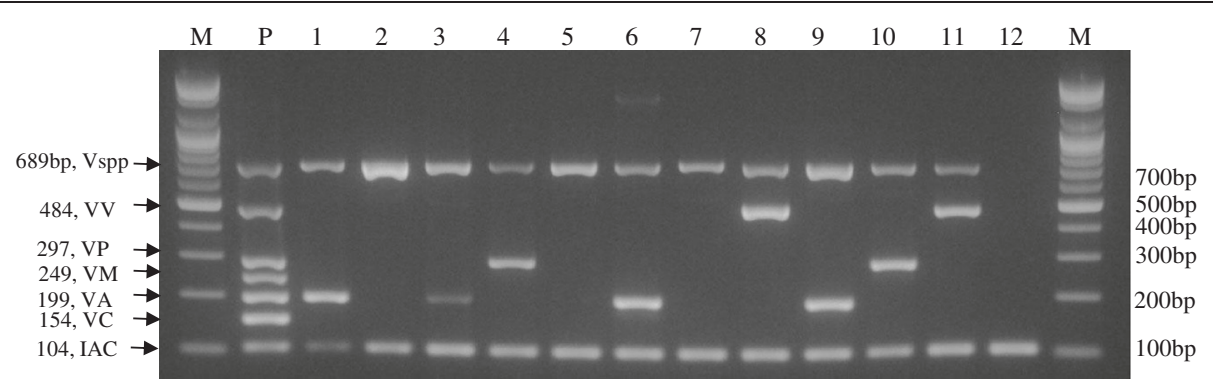

Fig. 2 Vibrio multiplex PCR results with genomic DNAs of representative Vibrio isolates from the south seashore areas in Korea. M: 100-bp DNA ladder, P: Mixture of Vibrio cholerae KCDC 13589, V. alginolyticus ATCC 17749, V. mimicus ATCC 33653, V. parahaemolyticus ATCC 27969 , V. vulnificus ATCC 33815, lane 1: Geoje-5, lane 2: Geoje -6, lane 3: Jinhae seawater-3, lane 4: Jinhae sand-water-3, lane 5: Jinhae sand-water-2, lane 6: Chungmu-1, lane 7: Chungmu-2, lane 8: MFDS Yeosu-3, lane 9: Busan-7, lane 10: Busan-6, lane 11: Busan-1, lane 12: no template. Vspp, Vibrio genus; W, V. vulnificus; VP, V. parahaemolyticus; VM, V. mimicus; VA, V. alginolyticus; VC, V. cholerae

Vibrio species in the food and clinical microbiology fields. These methods aim to overcome the disadvantages of phenotype-based biochemical identification and to allow for reliable identification [8].

One of the impacts of our study on the development of a novel Vibrio multiplex PCR is that it provides an example of how to utilize comparative genomics with a limited genome database for practical application in diagnostics in food safety and clinical microbiology, particularly for the accurate identification of pathogenic bacteria. As shown in Table 5, our selected genetic marker genes were derived from comparative genomics using 30 genome sequences belonging to 19 Vibrio species, which are regarded as representative of these Vibrio species. We demonstrated the substantial potential of a limited bacterial genome database and bioinformatics technology in the diagnosis of human pathogens. In designing our Vibrio multiplex PCR, the selection of target Vibrio species was based on the reported number of human Vibrio infections and by the availability of genome sequences in the NCBI database, including $V$. mimicus and $V$. alginolyticus, which were ranked high among human infections caused by pathogenic Vibrio species $[1,4,9,38]$. In addition, this Vibrio multiplex PCR method can be complemented or modified by the addition or subtraction of primer sets based on the availability of genome sequences or on the clinical significance of Vibrio species.

Our approach using an improved multiplex PCR assay along with comparative genomics for Vibrio identification differentiates our study from previously reported PCR methods. Recently, many Vibrio multiplex PCR assays have been reported for the identification of the major pathogenic Vibrio species; however, these multiplex PCR assays do not provide the diagnostic level required to be inclusive of the Vibrio genus [3, 21-26]. Our Vibrio multiplex PCR assay consists of two diagnostic levels for 1) the Vibrio genus and 2) five pathogenic Vibrio species. The reliable diagnosis of the Vibrio genus is important because the Vibrio genus consists of more

Table 4 Identification results of representative Vibrio isolates between Vibrio multiplex PCR and MALDI-TOF MS system

\begin{tabular}{|c|c|c|c|}
\hline \multicolumn{2}{|c|}{ Identification of Vibrio isolates by Vibrio multiplex PCR } & \multicolumn{2}{|c|}{ Identification of Vibrio isolates by MALDI-TOF MS analysis } \\
\hline Lane No. in Fig. $2^{a}$ & Description & MALDI-TOF MS results & $\log$ score value ${ }^{\mathrm{b}}$ \\
\hline 1 & Vibrio alginolyticus & Vibrio alginolyticus DSM $2171 \mathrm{~T}$ & 1.709 \\
\hline 2 & Vibrio spp. & Vibrio mytili DSM 19137 T & 1.721 \\
\hline 3 & Vibrio alginolyticus & Vibrio alginolyticus DSM $2171 \mathrm{~T}$ & 1.812 \\
\hline 4 & Vibrio parahaemolyticus & Vibrio parahaemolyticus DSM 11058 & 1.975 \\
\hline 5 & Vibrio spp. & Vibrio mytili DSM 19137 T & 1.756 \\
\hline 6 & Vibrio alginolyticus & Vibrio alginolyticus DSM $2171 \mathrm{~T}$ & 1.761 \\
\hline 7 & Vibrio spp. & Vibrio fortis DSM $19133 \mathrm{~T}$ & 1.933 \\
\hline 8 & Vibrio vulnificus & Vibrio vulnificus DSM 10143 T & 2.06 \\
\hline 9 & Vibrio alginolyticus & Vibrio alginolyticus CCM 2578 T & 1.755 \\
\hline 10 & Vibrio parahaemolyticus & Vibrio parahaemolyticus DSM 11058 & 1.951 \\
\hline 11 & Vibrio vulnificus & Vibrio vulnificus CCUG 38429 & 2.144 \\
\hline
\end{tabular}

${ }^{\mathrm{a}}$ The number indicate lane number of Vibrio isolates in Fig. 2

${ }^{\mathrm{b}}$ Isolates identification (log) score values, $>2.0$ indicated species-level, between 1.7 and 2.0 indicated genus level identification, respectively 
Table 5 Genome sequences of Vibrio strains used in this study ${ }^{a}$

\begin{tabular}{|c|c|c|c|c|c|}
\hline Strain & Reference sequence ${ }^{b}$ & Genome size (Mb) & $\begin{array}{l}\text { Number of genes used } \\
\text { in this study (on ffn file) }\end{array}$ & Release Date & $\begin{array}{l}\text { Status of genome } \\
\text { project }\end{array}$ \\
\hline $\begin{array}{l}\text { V. parahaemolyticus RIMD } \\
2210633\end{array}$ & NC_004603.1, NC_004605.1 & 5.17 & 4832 & 2003/03/05 & Completed \\
\hline $\begin{array}{l}\text { V. cholerae O1 biovar El Tor } \\
\text { str. N16961 }\end{array}$ & NC_002505.1, NC_002506.1 & 4.03 & 3834 & $2000 / 06 / 14$ & Completed \\
\hline V. cholerae M66 & NC_012578.1, NC_012580.1 & 3.94 & 3693 & $2009 / 04 / 20$ & Completed \\
\hline V. cholerae MJ 1236 & NC_012667.1, NC_012668.1 & 4.24 & 3772 & 2009/05/04 & Completed \\
\hline V. cholerae $\mathrm{O} 395$ & NC_009456.1, NC_009457.1 & 4.13 & 3998 & 2007/05/08 & Completed \\
\hline V. vulnificus CMCP6 & NC_004459.3, NC_004460.2 & 5.13 & 4433 & $2002 / 12 / 22$ & Completed \\
\hline V. vulnificus MO6 $24 \mathrm{O}$ & NC_014966.1, NC_014965.1 & 5.01 & 4562 & $2011 / 01 / 24$ & Completed \\
\hline V. vulnificus YJ016 & NC_005140.1, NC_005139.1 & 5.26 & 5023 & 2003/10/01 & Completed \\
\hline V. fischeri MJ11 & NC_011184.1, NC_011186.1 & 4.5 & 4039 & $2008 / 08 / 28$ & Completed \\
\hline V. fischeri ES114 & NC_006841.2, NC_006840.2 & 4.27 & 3817 & 2005/02/11 & Completed \\
\hline V. anguillarum 775 & NC_015637.1, NC_015633.1 & 4.05 & 3732 & 2011/06/09 & Completed \\
\hline V. species Ex25 & NC_013456.1, NC_013457.1 & 5.09 & 4518 & 2009/11/03 & Completed \\
\hline V. harveyi ATCC BAA-1116 & NC_009783.1, NC_009784.1 & 6.06 & 6041 & $2007 / 08 / 28$ & Completed \\
\hline V. splendidus LGP32 & NC_011753.2, NC_011744.2 & 4.97 & 4432 & $2008 / 12 / 17$ & Completed \\
\hline V. alginolyticus $12 \mathrm{G} 01$ & NZ_CH902589 NZ_CH902598 & 5.16 & & $2006 / 04 / 06$ & Scaffolds \\
\hline V. mimicus MB-451 & $\begin{array}{l}\text { NZ_ADAF01000001 } \\
\text { NZ_ADAF01000003C }\end{array}$ & 4.31 & & $2009 / 10 / 23$ & Scaffolds \\
\hline
\end{tabular}

${ }^{a}$ The information in this table was updated in June 2012. Fourteen genome shotgun sequences of Vibrio strains are not listed in this table

${ }^{b}$ Completed genome sequences were obtained from genomes on the NCBI site (ftp://ftp.ncbi.nlm.nih.gov/genomes/) in December 2011

${ }^{c}$ The genome sequences of $V$. alginolyticus and $V$. mimicus were not completed. Scaffolds sequence data were obtained from the NCBI site and used for comparative genomics in this study

than 30 species, including 11 reported human pathogenic Vibrio species $[1,4]$. In addition to being able to diagnose the Vibrio genus, this multiplex PCR allows for the identification of the five major pathogenic Vibrio species: $V$. cholerae, $V$. parahaemolyticus, $V$. vulnificus, $V$. alginolyticus and $V$. mimicus. The ability to simultaneously identify the five major pathogenic Vibrio species in single reaction is valuable in the clinical and food microbiology fields in that it provides informative diagnostics at the Vibrio genus level as well as at the species level. Another distinct feature of our Vibrio multiplex PCR is the novel genetic markers for the five major pathogenic Vibrio species that were derived through the use of comparative genomics. These genetic markers are different from those in other previous reports in which marker gene selection was based on the functional qualities of the proteins encoded by their virulence-related/ regulatory genes, or on phylogenetic classification of the housekeeping genes within Vibrio spp. [3, 8, 12-22]. We sought to acquire highly specific genetic marker genes for the diagnosis of Vibrio species by considering their presence/absence in the Vibrio genus and other closely related bacteria. We also considered the variable/conserved regions (sequence variation) in the genetic marker genes within Vibrio species. Furthermore, our screening approach for novel genetic markers was based purely on gene sequence comparisons using comparative genomics and was therefore not tied to the functions of genes and consequently the selected genetic markers were hypothetical proteins or proteins with other functions, as presented in Table 2. To give more objective validation on the presence of our screened genetic marker genes in each target Vibrio species (genus), each marker gene was compared and confirmed with each available Vibrio genome sequence of NCBI microbial genome database (Additional file 3). All each marker gene of Vibrio multiplex PCR was present in all available Vibrio genome sequences of NCBI microbial genome database (Complete Genome, Chromosome, Scaffold levels).

While screening marker genes of the Vibrio genus present in all Vibrio genome sequences (core genome of Vibrio genus), most of the Vibrio genes were eliminated. Ultimately, we were left with two genes specific to the Vibrio genus, despite the fact that they are present not only in Vibrio spp., but also in closely related bacteria: recombinase A, C2694352-2693309 [GenBank: NC_004603.1] and ATP synthase subunit alpha (atpA), C3281450-3279879 [GenBank: NC_004603.1] of $V$. parahaemolyticus RIMD 2210633. Interestingly, the atp $A$ gene has already been reported in multiplex PCR assays for Vibrio species [3]. This supports the reliability of our genetic marker screening procedure using comparative genomics. Also, based on our 
screening results, we noted that the atp $A$ gene is a more useful genetic marker for the Vibrio genus than for the Vibrio species.

The authors acknowledge that a more comprehensive panel of Vibrio strains will be required for the validation of this multiplex PCR. However, our study is extensive in that a large sample set of Vibrio isolates was sampled from seashore areas in Korea by MFDS, BMIHE and our laboratory. A total of 117 strains were evaluated by multiplex PCR and all isolates were determined to be Vibrio spp., as described in Table 3 and Fig. 2. Interestingly, while 94 isolates were identified as V. parahaemolyticus, $V$. vulnificus and $V$. alginolyticus, no isolates of $V$. cholerae or $V$. mimicus, which are considered to be more closely related to each other that to other Vibrio species [35, 37], were found. The results of the multiplex PCR assay that identified non- $V$. cholerae from seashore environmental samples are identical and comparable with those from a Vibrio monitoring study in live oysters by DePaola et al. [6], thereby supporting the specificity of our Vibrio multiplex PCR. Lastly, 23 isolates from among the 117 were identified as Vibrio spp., but were not among the 5 target Vibrio species used in this multiplex PCR, suggesting more informative diagnostics results with respect to other Vibrio species. Also, the identification of the representative Vibrio isolates using MALDI-TOF MS additionally supported the reliability of this Vibrio multiplex PCR as shown in Table 4.

\section{Conclusions}

The present study selected novel genetic marker genes for the Vibrio genus and five other Vibrio species using comparative genomics and developed a sextuplet multiplex PCR assay using designed primer sets that allows for informative identification of Vibrio, thereby enabling rapid and specific diagnostics. We utilized this Vibrio multiplex PCR to demonstrate its discriminative ability for the Vibrio genus and each of five major pathogenic Vibrio species through the evaluation of Vibrio strains and isolates. However, despite the fact that additional validation will be needed with various Vibrio strains in order to establish the reliability of this Vibrio multiplex PCR, we suggest that our results with respect to the reliable performance of this assay should be of sufficient impact to recommend application of the assay as a useful diagnostic for pathogenic Vibrio species.

\section{Methods}

\section{Bacterial strains}

The Vibrio strains used in this study were collected from the American Type Culture Collection (ATCC), the Korean Culture Center of Microorganisms (KCCM), and the National Culture Collection for Pathogens (NCCP) of Korea as shown in Table 1. The Vibrio strains were inoculated in tryptic soy broth (BD, Sparks, MD, USA) containing $3 \% \mathrm{NaCl}$ and incubated using the recommended culture conditions for genomic DNA extraction. Various non-Vibrio type strains, including food-borne pathogens and other closely related bacterial type strains, were collected from the ATCC and NCCP, and incubated using the recommended culture conditions.

\section{Genome sequences of Vibrio species}

Genome sequences and their Vibrio strain references used in this study are shown in Table 5 (14 uncompleted genome shotgun sequences of Vibrio strains are not shown in this table). A total of 14 completed genome sequences and 16 whole genome shotgun sequences (Scaffolds or contigs) of Vibrio strains, including $V$. parahaemolyticus, $V$. cholerae, $V$. vulnificus, $V$. alginolyticus and $V$. mimicus, were obtained from the National Center for Biotechnology Information (NCBI) web site [38] between December 2011 and April 2012.

\section{Comparative genomics for screening each Vibrio species- specific gene sequence}

One representative genome sequence of each target Vibrio species was used for species-specific gene screening. These included: Vibrio parahaemolyticus RIMD 2210633 [GenBank: NC_004603.1, NC_004605.1] [36], Vibrio cholerae O1 biovar EI Tor str. N16961 [GenBank: NC_002505.1, NC_002506.1] [39], Vibrio vulnificus YJ016 [GenBank: NC_005139.ffn, NC_005140.ffn], Vibrio alginolyticus 12G01 [GenBank: NZ_CH902589 NZ_CH902598] and Vibrio mimicus MB-451 [GenBank: NZ_ADAF01000001 NZ_ ADAF01000003] [37]. Scaffold sequences of $V$. alginolyticus and $V$. Mimicus, whose genome projects are not completed, were used for comparative genomics. To screen a specific gene (or DNA sequence) from each Vibrio species, the coding region sequences (ffn file) of each Vibrio species (target-Vibrio species) were compared against the genomic DNA sequences (fna file), which consist of Vibrio species excluding the genome sequence of the particular target Vibrio species, using the BLAST program (version 2.2.13) [40]. Based on BLAST analysis, we selected genes for each target Vibrio species that had low homology scores relative to the genomes of other Vibrio species and then recompared them against the non-redundant (nr) DNA sequence NCBI database. Final candidate genes of each Vibrio species-specific were used for the design of primer sets.

\section{Comparative genomics for screening Vibrio genus-specific gene sequences}

For screening Vibrio genus-specific genes, the coding region sequences of Vibrio parahaemolyticus RIMD 2210633 [GenBank: NC_004603.1, NC_004605.1] were used as the 
representative genome sequence of the Vibrio genus. The coding region sequences of Vibrio parahaemolyticus RIMD 2210633 (ffn file) were compared to each genome sequence of $V$. cholerae, $V$. vulnificus, $V$. mimicus and $V$. alginolyticus in order using the BLAST program and highly homologous genes expected to be present in all Vibrio species were screened. The screened genes were compared against the $\mathrm{nr}$ database and the microbial genomic database (representing complete and draft genome databases of microbes, respectively) on the NCBI web site. Vibrio genus-specific genes, which resulted in low homology (low sequence match) [33] considering their matched size and score of BLAST output, were selected for the design of Vibrio genus-specific primer sets.

\section{Genomic DNA extraction}

Cultured media from each bacterial strain was harvested in microtubes and the genomic DNA of each strain was extracted using the Genomic DNA extraction kit for bacteria (iNtRON Biotechnology, Seoul, Korea), according to the manufacturers instructions. Genomic DNA concentration was measured using a UV-spectrophotometer (Model UV-1700, Shimadzu, Tokyo, Japan) and genomic DNAs with spectrophotometric ratios of 1.8 to $2.0\left(\mathrm{~A}_{260} / \mathrm{A}_{280}\right)$ were used. Genomic DNAs were stored at $-20{ }^{\circ} \mathrm{C}$.

\section{Primer construction and PCR conditions}

Primer sets, expected to be specific to the Vibrio genus and/or species, were designed from each of the screened candidate genes and were evaluated using genomic DNAs of Vibrio and other type strains listed in Table 1. PCR amplifications were carried out with $200 \mu \mathrm{M}$ of each dNTP, 0.5 unit of Ex Taq DNA polymerase (TaKaRa Bio Inc., Shiga, Japan), 1× Ex Taq buffer, 25 ng of template DNA and the adjusted concentration of each primer in a final reaction volume of $25 \mu \mathrm{l}$. PCR amplification was performed in a thermocycler (Model PC 808, ASTEC, Fukuoka, Japan) with an initial denaturation at $94{ }^{\circ} \mathrm{C}$ for $5 \mathrm{~min}$, followed by 25 cycles of $94{ }^{\circ} \mathrm{C}$ for $30 \mathrm{~s}$, $60{ }^{\circ} \mathrm{C}$ for $30 \mathrm{~s}, 72{ }^{\circ} \mathrm{C}$ for $30 \mathrm{~s}$, finishing with a final extension at $72{ }^{\circ} \mathrm{C}$ for $10 \mathrm{~min}$ and storage at $4{ }^{\circ} \mathrm{C}$ thereafter. Amplified products were electrophoresed on a $3 \%$ agarose gel in $0.5 \times$ Tris-acetate-EDTA buffer, stained with ethidium bromide, visualized under UV-irradiation and photographed with a digital camera (Model COOLPIX 4300, Nikon, Tokyo, Japan).

\section{Multiplex PCR of Vibrio and construction of the internal amplification control (IAC)}

The multiplex PCR was designed to include six sets of screened primers, which targeted the Vibrio genus, $V$. parahaemolyticus, $V$. cholerae, $V$. vulnificus, $V$. alginolyticus and $V$. mimicus. The sequences of these primer sets along with their concentrations are shown in Table 2. In contrast to the single PCR reactions, one unit of Ex Taq DNA polymerase and $3 \mathrm{pg}$ (around $10^{6}$ copies) of IAC template were used in a single multiplex PCR reaction. The IAC template was constructed using the sequence of the target gene, c1155272-1154856 [GenBank: NC_004605.1] in Vibrio parahaemolyticus. A primer set was designated as VP c1155272 IAC F (5'- AGCTTAT TGGCGGTTTCTGTCGG CTACACCGTCGGCAGTG TGT -3') and VP c1155272 IAC R (5'- CGCAAGAC CAAGAAAAGCCGTC CTAGTGGCGTTTCGGAAA C $-3^{\prime}$ ), which were flanked with the primer sequence of $1155272 \mathrm{~F}-\mathrm{R}$ at the 5 ' end, resulting in amplification of a 104-bp DNA fragment including the partial gene sequence of c1155272-1154856 of $V$. parahaemolyticus. The amplified DNA fragment was inserted into pGEM-T Easy Vector (Promega Corporation, Madison, WI) to generate the IAC template plasmid enabling the amplification of the $104 \mathrm{bp}-\mathrm{PCR}$ product with this internal control sequence by the $1155272 \mathrm{~F}-\mathrm{R}$ primer set as a positive control for the Vibrio multiplex PCR.

\section{Limit of detection (LOD) and multi-detection ability for Vibrio species}

For the LOD experiment using the Vibrio multiplex PCR, the quantity of Vibrio genomic DNAs was calculated as the copy number by genome size. As an example, for Vibrio parahaemolyticus (genome size of Vibrio parahaemolyticus RIMD 2210633: $5.17 \mathrm{Mb}$ ), $56.7 \mathrm{ng}$ was considered to be $10^{7}$ copies of genomic DNA and was diluted from $10^{6}$ to $10^{0}$ copies per microliter. Diluted genomic DNA was added from $5 \times 10^{6}$ copies to $5 \times 10^{0}$ copies in each reaction and $5 \mu \mathrm{l}$ of the $25 \mu \mathrm{l}$-PCR products was loaded for $3 \%$ agarose gel electrophoresis. The multi-detection ability of the Vibrio multiplex PCR was also evaluated with various combinations of genomic DNAs from the five $V i b$ rio species (1 ng per each Vibrio species sample).

\section{Collection and isolation of Vibrio isolates from the south} seashore areas in Korea and evaluation of multiplex PCR The isolated strains of $V$. parahaemolyticus and $V$. vulnificus sampled from the seashore of Busan and Yeosu in South Korea were obtained from BMIHE and MFDS in South Korea, respectively. Other Vibrio strains were isolated from 4 south seashore areas, Busan, Geoje, Jinhae, and Chungmu, in Korea, using isolation methods recommended by the bacteriological analytical manual from the FDA [8]. In brief, samples from each local area were kept at 7 to $10{ }^{\circ} \mathrm{C}$ until delivered to the laboratory. Then, $25 \mathrm{~g}$ (or $50 \mathrm{ml}$ of liquid) of sample was placed into a stomacher bag and $225 \mathrm{ml}$ of phosphate buffered saline (PBS) was added. Samples were homogenized for $1 \mathrm{~min}$ at maximum RPM using a stomacher (Seward Stomachers ${ }^{\oplus} 400$ Circulator, Manchester, UK). One milliliter of homogenized sample was inoculated into $10 \mathrm{ml}$ 
of alkaline peptone water (APW) and was incubated overnight at $35{ }^{\circ} \mathrm{C}$. An inoculating loop was used to streak bacteria from the top of the APW onto thiosulfate-citrate-bile salt-sucrose Agar (TCBS agar, BD, Sparks, MD, USA) and the plate was incubated overnight at $35{ }^{\circ} \mathrm{C}$. Vibrio positive colonies, which were yellow or green to bluish-green colonies on TCBS agar, were sampled and cultured in TSB media containing $3 \%$ $\mathrm{NaCl}$ for isolation of stock or genomic DNA extraction allowing for use in multiplex PCR.

\section{PCR product sequencing}

Each amplified PCR product was purified from agarose gels using the QIAquick Gel Extraction Kit (Qiagen $\mathrm{GmbH}$, Hilden, Germany) and by QIAquick PCR Purification Kit (Qiagen). The sequencing of purified PCR products was performed using an automated DNA sequencer (Applied Biosystems, Foster City, CA, USA) using the forward and reverse primers used in the Vibrio multiplex PCR. The sequencing data was compared with the known targeted gene sequences which were originally used for specific-primer design for each Vibrio species.

\section{MALDI-TOF MS analysis}

For the identification of Vibrio isolates by means of MALDI-TOF MS, an individual colony were deposited directly on a target polished steel microscout target plate (MSP 96; Bruker Daltonik GmbH, Bremen, Germany) overlaid with $1 \mu \mathrm{l}$ of $70 \%$ formic acid and $1 \mu \mathrm{l}$ of $\alpha$-cyano4-hydroxycinnamic acid matrix solution in acetonitrile : water : trifluoro acetic acid (TFA) (ratio 50:47.5:2.5, v/v) and then air-dried. After crystallization, measurements were performed on a microflex LT bench-top mass spectrometer (Bruker Daltonik $\mathrm{GmbH}$ ) with a smart beam laser. The parameter conditions were as follows: ion source 1, $20.0 \mathrm{kV}$; ion source 2, $18.2 \mathrm{kV}$; lens, $6.0 \mathrm{kV}$; initial laser power; $25 \%$; maximal laser power; $35 \%$. Ionization was performed with laser irradiation. Raw spectra data were imported into Biotyper software 3.0 (Bruker Daltonik $\mathrm{GmbH}$ ). Mass spectra were collected within a mass range of $2000-20,000 \mathrm{~m} / \mathrm{z}$, with 1200 satisfactory laser shots in 240 shot steps. Prior to analysis, the reference strain Escherichia coli DH5 $\alpha$ was used as a standard for calibration and as reference for quality control. Each sample was matched to a reference library in the Biotyper software database, which contains spectra of approximately 5627 species.

\section{Additional files}

Additional file 1: (Figure) Limit of detection (LOD) results of Vibrio multiplex PCR. Panel (A) 25 cycles, Panel (B) 30 cycles, loaded amount of PCR product was $5 \mu$ from $25 \mu \mathrm{l} \mathrm{PCR} \mathrm{product.} \mathrm{M:} 100$ bp ladder, $1: 5 \times$ $10^{6}$ copies, $2: 5 \times 10^{5}$ copies, $3: 5 \times 10^{4}$ copies, $4: 5 \times 10^{3}$ copies, $5: 5 \times 10^{2}$ copies, 6: $5 \times 10^{1}$ copies, 7: $5 \times 10^{0}$ copies, 8: No Template (NT); Vspp, Vibrio genus; VC, Vibrio cholerae KCDC 13589; VA, V. alginolyticus ATCC 17749; VM, V. mimicus ATCC 33653; VP, V. parahaemolyticus ATCC 27969; W, V. Vulnificus ATCC 33815. (PPTX $321 \mathrm{~kb}$ )

Additional file 2: (Figure) Results of Vibrio multiplex PCR with genomic DNA combinations from Vibrio species. M: 100-bp ladder, 1: VC VA, 2: VC VM, 3: VC VP, 4: VC W, 5: VA VM, 6: VA VP, 7: VA W, 8: VM VP, 9: VM W, 10: VP W, 11: VC VA VM, 12: VC VM VP, 13: VC VM W, 14: VC VA W, 15: VA VM VP, 16: VA VM W, 17: VA VP W, 18: VM VP W, 19: VC VA VM VP, 20: VC VM VP W, 21: VA VM VP W, 22: VC VA VP W, 23: VC VA VM VP W, 24: NT. Vspp, Vibrio genus; VC, Vibrio cholerae KCDC 13589; VA, V. alginolyticus ATCC 17749; VM, V. mimicus ATCC 33653; VP, V. parahaemolyticus ATCC 27969; W, V. Vulnificus ATCC 33815. (PPTX $382 \mathrm{~kb})$

Additional file 3: (Tables) Presences of the six marker genes used for the Vibrio multiplex PCR in each genome of NCBI microbial genome database. The lists of genomes (Column A) were obtained from NCBl genome database (http://www.ncbinlm.nih.gov/genome/browse/). The matched genome sequence (Column C), ID and position of sequence (Column D) are BLAST results at microbial genome BLAST web site in NCBI (http://blast.ncbi.nlm.nih.gov/Blast.cgi?PAGE_TYPE=BlastSearch\&BLAST_ SPEC=MicrobialGenomes). (XLSX 78 kb)

\section{Competing interests}

The authors declare that they have no competing interests.

\section{Authors' contributions}

Conceived and designed the experiment: HJK, HYK. Performed the experiments: JOR, SYL, HJK, ESK. Contributed reagents/materials/analysis tools: JOR, SYL, HJK, HYK. Wrote the paper: HJK, HYK. All authors read and approved the final manuscript.

\section{Acknowledgment}

This work was supported by a grant from the Agenda Program (PJ009237) of the Rural Development Administration in the Republic of Korea.

Received: 8 January 2015 Accepted: 19 October 2015

Published online: 26 October 2015

\section{References}

1. Janda JM, Powers C, Bryant RG, Abbott SL. Current perspectives on the epidemiology and pathogenesis of clinically significant Vibrio spp. Clin Microbiol Rev. 1988;1:245-67.

2. Scallan E, Hoekstra RM, Angulo FJ, Tauxe RV, Widdowson MA, Roy SL, et al. Foodborne illness acquired in the united states-major pathogens. Emerg Infect Dis. 2011;17:7-15.

3. Izumiya H, Matsumoto K, Yahiro S, Lee J, Morita M, Yamamoto S, et al. Multiplex PCR assay for identification of three major pathogenic Vibrio spp., Vibrio cholerae, Vibrio parahaemolyticus, and Vibrio vulnificus. Mol Cell Probes. 2011;25:174-6.

4. Thompson FL, lida T, Swings J. Biodiversity of vibrios. Microbiol Mol Biol Rev. 2004;68:403-31.

5. Iwamoto M, Ayers T, Mahon BE, Swerdlow DL. Epidemiology of seafoodassociated infections in the United States. Clin Microbiol Rev. 2010;23:399-411.

6. DePaola A, Jones JL, Woods J, Burkhardt 3rd W, Calci KR, Krantz JA, et al. Bacterial and viral pathogens in live oysters: 2007 United States market survey. Appl Environ Microbiol. 2010;76:2754-68.

7. Espiñeira M, Atanassova M, Vieites JM, Santaclara FJ. Validation of a method for the detection of five species, serogroups, biotypes and virulence factors of Vibrio by multiplex PCR in fish and seafood. Food Microbiol. 2010;27:122-31.

8. Kaysner CA, DePaola A: Vibrio cholerae, V. parahaemolyticus, V. vulnificus, and other Vibrio spp., Bacteriological analytical manual, 8th edition, revision A, 1998. [http://www.fda.gov/food/foodscienceresearch/laboratorymethods/ ucm070830.htm]

9. Centers for Disease Control and Prevention (CDC), National Cholera and Vibriosis Surveillance: Cholera and Other Vibrio Illness Surveillance (COVIS) Annual Summary, 2012. [http://www.cdc.gov/nationalsurveillance/choleravibrio-surveillance.html]

10. World Health Organization (WHO). Cholera, 2013. Wkly Epidemiol Rec. 2014;89:345-56 [http://www.who.int/cholera/statistics/en/]. 
11. Jones JL, Hara-Kudo Y, Krantz JA, Benner RA, Smith AB, Dambaugh TR, et al. Comparison of molecular detection methods for Vibrio parahaemolyticus and Vibrio vulnificus. Food Microbiol. 2012;30:105-11.

12. Dick MH, Guillerm M, Moussy F, Chaignat CL. Review of two decades of cholera diagnostics - How far have we really come? PLoS Negl Trop Dis. 2012;6:e1845.

13. Nordstrom JL, Vickery MCL, Blackstone GM, Murray SL, DePaola A. Development of a multiplex real-time PCR assay with an internal amplification control for the detection of total and pathogenic Vibrio parahaemolyticus bacteria in oysters. Appl Environ Microbiol. 2007;73:5840-7.

14. Nishibuchi M, Kaper JB. Nucleotide sequence of the thermostable direct hemolysin gene of Vibrio parahaemolyticus. J Bacteriol. 1985;162:558-64.

15. Bej AK, Patterson DP, Brasher CW, Vickery MCL, Jones DD, Kaysner CA. Detection of total and hemolysin-producing Vibrio parahaemolyticus in shellfish using multiplex PCR amplification of th, tdh and trh. J Microbiol Meth. 1999;36:215-25.

16. Hill WE, Keasler SP, Trucksess MW, Feng P, Kaysner CA, Lampel KA. Polymerase chain reaction identification of Vibrio vulnificus in artificially contaminated oysters. Appl Environ Microbiol. 1991;57:707-11.

17. Panicker G, Bej AK. Real-Time PCR detection of Vibrio vulnificus in oysters: comparison of oligonucleotide primers and probes targeting vvhA. Appl Environ Microbiol. 2005;71:5702-9.

18. Kim YB, Okuda J, Matsumoto C, Takahashi N, Hashimoto S, Nishibuchi M. Identification of Vibrio parahaemolyticus strains at the species level by PCR targeted to the toxR gene. J Clin Microbiol. 1999;37:1173-7.

19. Takahashi H, Hara-Kudoa Y, Miyasaka J, Kumagaid S, Konuma H. Development of a quantitative real-time polymerase chain reaction targeted to the tox $R$ for detection of Vibrio vulnificus. J Microbiol Methods. 2005;61:77-85.

20. Matsumoto C, Okuda J, Ishibashi M, Iwanaga M, Garg P, Rammamurthy T, et al. Pandemic spread of an O3:K6 clone of Vibrio parahaemolyticus and emergence of related strains evidenced by arbitrarily primed PCR and toxRS sequence analyses. J Clin Microbiol. 2000;38:578-85.

21. Nhunga PH, Ohkusua K, Miyasakab J, Suna XS, Ezakia T. Rapid and specific identification of 5 human pathogenic Vibrio species by multiplex polymerase chain reaction targeted to dna gene. Diagn Microbiol Infect Dis. 2007:59:271-5.

22. Tarr CL, Patel JS, Puhr ND, Sowers EG, Bopp CA, Strockb NA. Identification of Vibrio isolates by a multiplex PCR assay and $r p o B$ sequence determination. J Clin Microbiol. 2007:45:134-40.

23. Bauer A, Rørvik LM. A novel multiplex PCR for the identification of Vibrio parahaemolyticus, Vibrio cholerae and Vibrio vulnificus. Lett Appl Microbiol. 2007:45:371-5.

24. Neogi SB, Chowdhury N, Asakura M, Hinenoya A, Haldar S, Saidi SM, et al. A highly sensitive and specific multiplex PCR assay for simultaneous detection of Vibrio cholerae, Vibrio parahaemolyticus and Vibrio vulnificus. Lett Appl Microbiol. 2010;51:293-300.

25. Vinothkumar K, Bhardwaj AK, Ramamurthy T. Triplex PCR assay for the rapid identification of 3 major Vibrio species, Vibrio cholerae, Vibrio parahaemolyticus, and Vibrio fluvialis. Diagn Microbiol Infect Dis. 2013;76:526-8.

26. Wang R, Huang J, Zhang W, Lin G, Lian J, Jiang L, et al. Detection and identification of Vibrio parahaemolyticus by multiplex PCR and DNA-DNA hybridization on a microarray. J Genet Genomics. 2011;38:129-35.

27. Dalmasso A, Civera T, Bottero MT. Multiplex primer-extension assay for identification of six pathogenic vibrios. Int J Food Microbiol. 2009;129:21-5.

28. Tracz DM, Backhouse PG, Olson AB, McCrea JK, Walsh JA, Ng LK, et al. Rapid detection of Vibrio species using liquid microsphere arrays and real-time PCR targeting the ftsZ locus. J Med Microbiol. 2007;56:56-65.

29. Kim HJ, Park SH, Kim HY. Genomic sequence comparison of Salmonella enterica serovar Typhimurium LT2 with Salmonella genomic sequences, and genotyping of Salmonellae by using PCR. Appl Environ Microbiol. 2006;72:6142-51.

30. Kim HJ, Park SH, Lee TH, Nahm BH, Chung YH, Seo KH, et al. Identification of Salmonella enterica serovar Typhimurium using specific PCR primers obtained by comparative genomics in Salmonella serovars. J Food Protect. 2006;69:1653-61.

31. Park SH, Kim HJ, Cho WH, Kim JH, Oh MH, Kim SH, et al. Identification of Salmonella enterica subspecies I, Salmonella enterica serovars Typhimurium, Enteritidis and Typhi using multiplex PCR. FEMS Microbiol Lett. 2009;301:137-46

32. Kim HJ, Park SH, Lee TH, Nahm BH, Kim YR, Kim HY. Microarray detection of food-borne pathogens using specific probes prepared by comparative genomics. Biosens Bioelectron. 2008;24:238-46.
33. NCBI BLAST web site [http://blast.ncbi.nlm.nih.gov/Blast.cgi?CMD=Web\& PAGE_TYPE=BlastHome]

34. Yeung PS, Boor KJ. Epidemiology, pathogenesis, and prevention of foodborne Vibrio parahaemolyticus infections. Foodborne Pathog Dis. 2004;1:74-88.

35. Davis BR, Fanning GR, Madden JM, Steigerwalt AG, Bradford Jr HB, Smith Jr $\mathrm{HL}$, et al. Characterization of biochemically atypical Vibrio cholerae strains and designation of a new pathogenic species, Vibrio mimicus. J Clin Microbiol. 1981;14:631-9.

36. Makino K, Oshima K, Kurokawa K, Yokoyama K, Uda T, Tagomori K, et al. Genome sequence of Vibrio parahaemolyticus: a pathogenic mechanism distinct from that of $V$. cholerae. Lancet. 2003;361:743-9.

37. Hasan NA, Grima CJ, Haleya BJ, Chunc J, Alame M, Taviania E, et al. Comparative genomics of clinical and environmental Vibrio mimicus. Proc Natl Acad Sci U S A. 2010;107:21134-9.

38. Genome database of the National Center for Biotechnology Information (NCBI) [http://www.ncbi.nlm.nih.gov/genome/]

39. Heidelberg JF, Eisen JA, Nelson WC, Clayton RA, Gwinn ML, Dodson RJ, et al. DNA sequence of both chromosomes of the cholera pathogen Vibrio cholera. Nature. 2000;406:477-83.

40. Altschul SF, Madden TL, Schäffer AA, Zhang J, Zhang Z, Miller W, et al. Gapped BLAST and PSI-BLAST: a new generation of protein database search programs. Nucleic Acids Res. 1997;25:3389-402.

\section{Submit your next manuscript to BioMed Central and take full advantage of:}

- Convenient online submission

- Thorough peer review

- No space constraints or color figure charges

- Immediate publication on acceptance

- Inclusion in PubMed, CAS, Scopus and Google Scholar

- Research which is freely available for redistribution 